\title{
Alimentação hospitalar: elementos para a construção de iniciativas humanizadoras
}

\section{Hospital food: elements to build humanizing initiatives}

\author{
Anete Araújo de Sousa' \\ Raquel Kuerten de Salles \\ Liana Ferraz Ziliotto2 \\ Ana Paula Aguiar Prudêncio² \\ Carla Adriano Martins ${ }^{3}$ \\ Cassiani Gotâma Tasca Pedroso $0^{3}$ \\ ${ }^{1}$ Núcleo de Pesquisa de Nutrição em Produção \\ de Refeições. Universidade Federal de Santa \\ Catarina. Campus Universitário, Trindade, \\ Florianópolis, SC, Brasil. \\ ${ }^{2}$ Curso de Nutrição, Núcleo de Pesquisa de \\ Nutrição em Produção de Refeições. Universidade \\ Federal de Santa Catarina. Campus Universitário, \\ Trindade, Florianópolis, SC, Brasil. \\ 3 Programa de Pós-Graduação em Nutrição. \\ Núcleo de Pesquisa de Nutrição em Produção \\ de Refeições. Campus Universitário, Trindade, \\ Florianópolis, SC, Brasil
}

Correspondência / Correspondence Anete Araújo de Sousa E-mail: anete@ccs.ufsc.br

\section{Resumo}

Objetivo: Identificar percepções de nutricionistas sobre iniciativas de humanização em alimentação e nutrição na atenção hospitalar. Métodos: Abordagem qualitativa, do tipo exploratória, através da técnica de grupos focais com auxílio de perguntas norteadoras e guia de entrevista. Participaram 23 nutricionistas de quatro hospitais de diferentes naturezas jurídicas, distribuídos em cinco grupos focais. Dados foram analisados através da técnica de análise de conteúdo. Resultados: Foram identificadas duas categorias: "Percepções da humanização na alimentação hospitalar" e "Iniciativas de humanização", evidenciando os seguintes elementos de análise: individualização do atendimento; educação nutricional; atendimento continuado pós-alta; melhoria nutricional e sensorial dos cardápios, conjugada à dimensão simbólica e convivial da alimentação; organização do serviço; formação permanente; sensibilização e valorização dos profissionais. Conclusão: As iniciativas de humanização destacadas revelaram aspectos das necessidades de usuários e profissionais. O conjunto dessas ações requer interação dos profissionais envolvidos, apoio institucional e individualização da atenção.

Palavras-chave: Alimentação Coletiva. Hospitais. Humanização da Assistência. Grupos Focais. Nutricionista. 


\section{Abstract}

Objectives: To identify perceptions of nutritionists about humanization initiatives in food and nutrition in hospital care. Methods: A qualitative exploratory approach, applying the focus groups technique with the aid of guiding questions and an interview guide was used. Twenty-three nutritionists, distributed into five focal groups, from four hospitals of different legal status participated in the study. Results: Content analysis enabled the construction of two categories: "Perceptions of the humanization of hospital meals" and "Humanization initiatives", providing evidence of the following elements of analysis: individualization of patient care; nutritional education; continuation of care after patient discharge; nutritional and sensorial improvement of meals; combination of the symbolic and social dimensions of food; organization of the service; educational actions; sensitizing and professional enhancement of staff. Conclusion: These initiatives of humanization revealed aspects of the needs of users and professionals. All these actions require interaction of the professionals involved, institutional support and individualized attention.

Key words: Collective Feeding. Hospitals. Humanization of Assistance. Focus Groups. Nutritionist.

\section{Introdução}

A alimentação hospitalar é reconhecida por sua relação com a melhoria do tratamento aos pacientes, em conjunto com outros cuidados de saúde. Esta consideração é particularmente importante em função da prevalência de desnutrição intra-hospitalar, ${ }^{1-4}$ aliada ao aumento da expectativa de vida e às mudanças nos perfis de morbidade da população. ${ }^{5}$

O estado nutricional do paciente hospitalizado é diretamente influenciado pela conduta nutricional implementada. Estudos demonstram que em geral os pacientes não ingerem alimentação suficiente para atender a suas necessidades nutricionais durante a hospitalização. ${ }^{6-11}$ Destaca-se ainda que há vulnerabilidade dos pacientes quando a qualidade no atendimento hospitalar se baseia somente na eficiência técnico-científica, focalizando apenas as doenças, os procedimentos e as tarefas. ${ }^{12}$ Entretanto, "o que determina se a tecnologia desumaniza, despersonaliza ou objetifica é a forma e o contexto como são utilizadas". ${ }^{13}$ Neste sentido, o setor hospitalar, ambiente caracterizado pela alta tecnologia, tem se preocupado com elementos de avaliação sobre o tipo de atendimento prestado, destacando-se a necessidade de ações com ênfase no cuidado humanizado. ${ }^{14,15}$ 
No Brasil, a Política Nacional de Humanização (PNH) caracteriza o cuidado humanizado como a oferta de atendimento de qualidade, articulando avanços tecnológicos com acolhimento e possibilitando a melhoria dos ambientes e das condições de trabalho dos profissionais. ${ }^{16} \mathrm{~A} \mathrm{PNH}$ propõe um conjunto de iniciativas e estratégias com o intuito de promover a qualidade do cuidado, respeitando os direitos do paciente, sua subjetividade e cultura, além de valorizar os diferentes sujeitos envolvidos no processo de produção de saúde. Deslandes,${ }^{17}$ ao realizar uma leitura crítica sobre a PNH, aponta três elementos que exigem maior reflexão e debate, como o uso da tecnologia no contexto da humanização, a comunicação entre os sujeitos e as condições estruturais para o trabalho dos profissionais.

Ayres $^{18}$ argumenta que, na humanização do atendimento, deve-se atentar para o cuidado em saúde e o efetivo compromisso com o ser humano, visando a uma interação simétrica entre os sujeitos na reorganização das práticas de atenção à saúde. Destaca ainda que é preciso que os profissionais "se responsabilizem em relação aos projetos de felicidade daqueles de cuja saúde cuidam", por isso a importância de relacionar aspectos técnicos com aspectos humanistas. ${ }^{19}$ Boff ${ }^{20}$ ressalta que "o que se opõe ao descuido e ao descaso é o cuidado. Cuidar é mais do que um ato; é uma atitude. Portanto, abrange mais que um momento de atenção, de zelo e de desvelo. Representa uma atitude de ocupação, preocupação, de responsabilização e de envolvimento afetivo com o outro."

Todavia, constatam-se na literatura científica poucas discussões sobre a humanização na alimentação hospitalar. Algumas propostas de ações e estratégias humanizadas para os cuidados em alimentação e nutrição foram levantadas por Poulain \& Saint-Sevin, ${ }^{21}$ Poulain, Jeanneau \& Tibère, ${ }^{22}$ Sousa \& Proença, ${ }^{23}$ Demário, Sousa \& Salles ${ }^{24}$ e Pedroso, Sousa \& Salles. ${ }^{25}$ Nas análises, esses estudos destacam que as refeições hospitalares podem estar ligadas, além das dimensões nutricionais e higiênico-sanitárias, ao conforto e acolhimento, valorizando os aspectos da convivialidade e as dimensões simbólicas e sensoriais.

Diante dessas considerações, o objetivo desta pesquisa foi identificar percepções de nutricionistas sobre iniciativas de humanização em alimentação e nutrição na atenção hospitalar.

\section{Método}

A pesquisa apresentou abordagem qualitativa, ${ }^{26}$ do tipo exploratória, utilizando a técnica dos grupos focais. ${ }^{27,28}$

Os grupos focais foram compostos por nutricionistas que atuavam em quatro hospitais do município de Florianópolis-SC, de diferentes naturezas jurídicas (3 hospitais públicos, sendo um federal e dois estaduais e um misto, com atendimento privado e filantrópico). A seleção desses hospitais foi intencional, pois neles havia o processo de implementação de instrumentos 
para qualificar o atendimento humanizado aos pacientes. Esta característica foi identificada, preliminarmente, em um estudo multicêntrico. ${ }^{29}$

Os grupos focais seguiram a metodologia proposta por Sobal. ${ }^{28}$ Foram realizados cinco grupos, com a participação de 23 nutricionistas. No hospital que contava com um número maior de profissionais (13 nutricionistas), estes foram distribuídos em dois grupos. Nos demais hospitais, os participantes formaram um grupo focal por instituição, composto por três ou quatro nutricionistas. Dois entrevistadores treinados conduziram os encontros, que foram realizados nas dependências dos hospitais.

Nos grupos focais, foram utilizadas duas perguntas norteadoras: "Como pode ser concebido um modelo de alimentação hospitalar humanizado?" e "Que atitudes humanizadoras são exequíveis para este tipo de atendimento?". Contou-se ainda com um guia de entrevistas, construído com base nos princípios da PNH ${ }^{16}$ e nos estudos de Poulain, Jeanneau \& Tibère, ${ }^{22}$ Sousa \& Proença, ${ }^{23}$ Demário, Sousa \& Salles ${ }^{24}$ e Pedroso, Sousa \& Salles ${ }^{25}$ (vide Apêndice).

Os encontros ocorreram no período de março a agosto de 2008, tendo duração média de uma hora e meia. As discussões foram registradas, gravadas e transcritas. Posteriormente, realizouse a autoconfrontação, através da qual cada participante recebeu cópia das transcrições para leitura e assinatura do material. Os relatos obtidos foram decompostos em unidades de registro e posteriormente agrupados em categorias analíticas. ${ }^{30}$ Este processo de categorização foi realizado em pares por pesquisadores. Por fim, as percepções que resultaram desse processo foram agregadas em duas categorias.

O projeto de pesquisa foi apreciado e aprovado pelo Comitê de Ética em Pesquisa com Seres Humanos (CEPSH) da Universidade Federal de Santa Catarina (UFSC), sob o no 153/2007, e os participantes assinaram o Termo de Consentimento Livre e Esclarecido (TCLE). A fim de assegurar o anonimato dos participantes, foi utilizada a identificação "Nut", seguida por algarismos cardinais (Nut 1; Nut 2...). Os hospitais foram identificados por HPr (hospital privado), e HPu (hospital público 1, 2 e 3).

\section{Resultados e discussão}

A análise dos relatos orientou a construção das categorias "Percepções sobre a humanização na alimentação hospitalar" e "Iniciativas de humanização", analisadas de forma conjunta. A percepção sobre humanização na alimentação hospitalar esteve relacionada à necessidade de atendimento individualizado ao usuário, centrado principalmente em suas expectativas e no cuidado que ultrapasse a visão de doença e de satisfação de necessidades físicas e nutricionais. O conforto e o respeito a seus hábitos alimentares, aliados aos serviços oferecidos e à segurança higiênico-sanitária, também foram aspectos relatados. 
Para esses nutricionistas, inseridos em contextos de humanização em seus ambientes de trabalho, a alimentação hospitalar refere-se não só às dimensões nutricionais do alimento ofertado, mas também às dimensões éticas, sensoriais, simbólicas e à qualificação do serviço.

Alimentação humanizada é o atendimento individualizado para todos, indistintamente de raça, de cor, convênio, vendo o indivíduo da mesma forma, e focando o lado humano. Não olhando o paciente como um objeto! (Nut1 - HPr).

Acho que é atender o paciente nutricionalmente, mas também o serviço [...]. É importante o serviço da copeira, a elaboração saborosa dos pratos, a apresentação [...], a atitude delas, arrumação da bandeja. Às vezes com um detalhe, você pode estar deixando esse trabalho, essa alimentação mais humanizada (Nut1 - HPu3).

Acho que não podemos esquecer que a alimentação humanizada passa pela questão da segurança higiênicosanitária e, enquanto nutricionistas, precisamos garantir esse tipo de qualidade (Nut1 - HPu2).

Observou-se nos relatos que existe a compreensão de que a humanização da alimentação hospitalar deve abranger distintas dimensões da alimentação. Esta percepção tem sido apontada por alguns autores. ${ }^{22-25}$ Todavia, segundo Garcia, ${ }^{31}$ os procedimentos higiênico-sanitários ainda consistem no aspecto mais valorizado pelos funcionários do serviço de alimentação.

A alimentação tem significados diferenciados para as pessoas. A refeição, em muitas culturas, é entendida como um espaço humanizador por excelência, possibilitando a reunião, a troca de ideias, as comemorações e ocupando espaço de destaque na vida familiar. ${ }^{32}$ Portanto, pensar na alimentação hospitalar como parte do cuidado ao paciente requer ir além da integração das qualidades e funções contempladas pelas dimensões nutricionais e higiênico-sanitárias. ${ }^{23}$

A internação caracteriza-se por um rompimento com os hábitos e o cotidiano dos pacientes, que por vezes são tratados como agentes passivos no que tange à alimentação hospitalar. ${ }^{33}$ Demário, Sousa \& Salles ${ }^{24}$ destacam que há conformação por parte dos pacientes, que se adaptam às normas impostas pelo hospital. Apesar dessa submissão às normas e seu impacto sobre a ingestão alimentar, os serviços de alimentação podem buscar estratégias para que a alimentação hospitalar seja mais próxima da realizada em casa. ${ }^{31}$

Neste sentido, as políticas de humanização nos hospitais podem incorporar a dimensão humana da alimentação, compreendendo o atendimento às individualidades de acordo com a história, as preferências e os hábitos alimentares de seus usuários. ${ }^{24}$ Deslandes ${ }^{17}$ argumenta que as diretrizes da PNH "serão inócuas se não forem valorizadas as expressões das expectativas e demandas dos próprios usuários-pacientes, reconhecendo sua autonomia e legitimidade simbólicas, cujas manifestações, a princípio, não são delegáveis a tradutores-intérpretes”. Assim, é responsabilidade dos profissionais atuantes na alimentação hospitalar a construção de iniciativas alinhadas com os 
princípios propostos pela $\mathrm{PNH},{ }^{16}$ que possibilitem a humanização do encontro entre profissional e paciente, bem como sua transformação em cuidado. ${ }^{19}$

Neste sentido, com base nas percepções e discussões apontadas, os nutricionistas elencaram iniciativas que consideraram relevantes para a melhoria de uma atuação mais humanizada. Tais iniciativas estão relacionadas ao atendimento das necessidades dos pacientes, como também às necessidades dos nutricionistas para prestar um atendimento humanizado, conforme destacado nos relatos que se seguem. Ao refletirem sobre as iniciativas de humanização para atender às necessidades dos pacientes, os nutricionistas apontaram a educação nutricional:

A gente já aproveita e trabalha a educação nutricional [...]. Apesar de que a gente gostaria de trabalhar mais (Nut3 - HPu2).

Pensar na educação nutricional como uma das iniciativas humanizadoras implica refletir sobre a concepção e a construção desta ação, bem como sua complexidade. Ao destacarem a educação nutricional como iniciativa humanizadora, os nutricionistas a referem possivelmente dentro da lógica de transmissão de informações, ${ }^{32}$ e não por meio de "troca e construção de saberes", conforme previsto na $\mathrm{PNH}^{16}$

Sousa \& Proença, ${ }^{23}$ discutindo a qualificação da alimentação hospitalar, recomendam o desenvolvimento de oficinas em grupo, explorando-se o conhecimento sobre os alimentos, as formas de preparo e a relação dos alimentos com a enfermidade, dentro de uma perspectiva de troca de conhecimentos. Além disso, os nutricionistas consideraram que a instalação de refeitórios nas unidades de internação seria mais uma iniciativa que poderia contribuir na aceitação das refeições e tornaria o ato alimentar mais prazeroso.

Seria interessante um refeitório onde os pacientes que podem se locomover fizessem a refeição ali e não na cama (Nut3 - HPu3).

Quando tinha os refeitórios nas unidades, percebíamos que os pacientes saíam do leito para realizar as refeições e isso fazia uma diferença muito grande no apetite e na aceitação (Nut3 - HPu2).

No mesmo sentido, realizar refeições em grupo pode favorecer o compartilhamento de impressões e experiências, ${ }^{33}$ reforçando a função convivial da alimentação. ${ }^{21}$ Essas interações são valorizadas pelos pacientes por possibilitarem um ambiente acolhedor e por tornarem a refeição um momento de troca. ${ }^{24}$

O cuidado no atendimento com responsabilidade e envolvimento afetivo, ${ }^{20}$ tanto dos copeiros como dos nutricionistas, foi considerado uma ação humanizada. 
Precisamos ser sensíveis ao paciente, à criança, à mãe [...] ainda mais com criança que precisa de todo aquele cuidado, aquela atenção, uma brincadeira [...], qualquer coisa para fazê-los sentir-se melhor (Nut3 - HPu2).

É atender as necessidades do paciente naquele momento. E necessidade não é só ir lá e entregar o alimento [...]. É ter carinho, é de afeto [...]. Às vezes é de um olhar, é de um sorriso, pra ele perceber que alguém naquele momento está preocupado com ele (Nut1 - HPr).

O processo de humanização da assistência busca o reencontro entre o acolhimento e o diálogo, contrapondo-se à massificação do atendimento ao ser humano. ${ }^{15,34-36}$ Neste caso, o acolhimento consiste em recurso essencial, por permitir a abertura à escuta do outro, destacando a importância da dimensão dialógica do encontro, não sendo o profissional um mero transmissor de discurso técnico-científico. ${ }^{19}$

Para um cuidado humanizado, apontou-se a possibilidade de escolha do tipo de refeição e preparações, caracterizando mais uma vez a individualização da atenção, como relatado anteriormente. Outras ações, como o atendimento de pedidos especiais pelos pacientes, a troca do horário da refeição, a escolha de um alimento de preferência e a opção pelo consumo de um lanche na hora do jantar, foram também referidas.

A gente percebe que quando a gente consegue atender um pedido do paciente com algo diferente, por exemplo, um pirão d'água do jeitinho que ele queria, ele come melhor [...], se anima (Nut6 - HPu1).

Se eles não estão comendo e dizem que não estão com vontade de comer "isso", a gente tenta fazer, principalmente paciente da oncologia, paciente queimado. Às vezes eles querem pizza, por exemplo, [...] a gente faz (Nut1 - HPu2).

O horário do jantar é flexível. Se o paciente quer jantar às $18 \mathrm{~h}$ ou $19 \mathrm{~h}$, existe essa possibilidade, sendo que o jantar é servido às 17 h, então isso também é individualizado (Nut1 - HPr).

A possibilidade de fazer adaptações individualizadas, ${ }^{37}$ além de aumentar o grau de satisfação dos pacientes, ainda potencializa a aceitação das dietas hospitalares. ${ }^{24}$ Deslandes, ${ }^{38}$ ao debater o artigo de Benevides \& Passos, ${ }^{39}$ argumenta que o desafio para a humanização dessas práticas está no reconhecimento da condição de pacientes como pessoas e sujeitos. Como pessoa, "passa pelo reconhecimento e respeito a distintos referenciais culturais. A noção de sujeito nos lança ao discurso ético da autonomia, das escolhas e decisões à luz das condições de gênero, posição social e etnia/raça".

Por outro lado, para atendimento das necessidades e expectativas dos pacientes, todos os nutricionistas ressaltaram que a prestação de um serviço qualificado está relacionada ao aumento do quadro de pessoal. Alegaram que a sobrecarga de trabalho dificulta a assistência humanizada. 
Faltam nutricionistas. A nossa equipe está cada vez mais reduzida, não só nutricionistas, mas a equipe toda. [...] Os nutricionistas mesmo, nós éramos em seis há um tempo atrás. Agora somos em três, a metade. Então, isso afeta o atendimento ao paciente (Nut1 - HPu3).

A gente tenta muitas vezes personalizar, apesar da nossa dificuldade pela quantidade pequena de nutricionistas. A gente não consegue dar atendimento individual para todos (Nut3 - HPu2).

Sabe-se que, além do número reduzido de profissionais, a complexidade das tarefas desempenhadas pelos nutricionistas consiste na dificuldade para a implementação de ações de cuidado alimentar e nutricional humanizado no hospital, com destaque para a falta de tempo, excesso de leitos por nutricionista e atividades administrativas. ${ }^{25}$ Sousa \& Proença ${ }^{23}$ argumentam que as Unidades de Alimentação e Nutrição devem contar com: estruturas de apoio para a formação de pessoal; desenvolvimento de sistemas informatizados que possam diminuir as atividades administrativas repetitivas; e renovação e manutenção preventiva e corretiva de áreas de trabalho, equipamentos e materiais. Além disso, a gestão do cuidado alimentar e nutricional necessitaria da redefinição das finalidades do trabalho do nutricionista, de produção de refeições e da terapia nutricional, não pressupondo a produção de refeições distanciada da clínica, nem o trabalho em clínica distanciado do alimento. Compreenderia, no entanto, uma relação de parceria que levaria a redescobrir novos espaços para o cuidado integral à saúde do ponto de vista alimentar e nutricional, englobando inclusive estratégias de interação e organização (horários de refeições adaptáveis aos usuários, refeitórios coletivos, comissão de cardápios, dentre outros).

Ainda, a atenção dos nutricionistas, mesmo após a alta hospitalar, e o interesse em atender da melhor forma possível, foram pontos levantados como iniciativas realizadas e que podem revelar a preocupação com a continuidade do atendimento.

Quando eles chegam em casa é que as dúvidas começam a surgir [...]. Então daí eles ficam bem à vontade pra ligar aqui pra gente, e pra conversarem também, [...] pra criar esse vínculo também (Nut2 - HPr).

Às vezes você vai perder uma hora, duas horas com o paciente, conversando com a mãe, ouvindo o que ela está falando [...] saber o que o paciente quer para poder se alimentar melhor, o que é melhor e o que não é. Então você vai atender, em todas as áreas da alimentação, o melhor possível (Nut.3 - HPu2).

$\mathrm{O}$ atendimento continuado está previsto na PNH. Além disso, as diretrizes para implantação dessa política especificam a necessidade de ampliação do diálogo, tanto entre profissionais, como entre profissionais e população atendida, reforçando o compromisso com o sujeito e seu coletivo, com as necessidades sociais de saúde, com a ambiência e com a melhoria das condições de atendimento e de trabalho ${ }^{16}$ 
Para os nutricionistas deste estudo, outra iniciativa apontada para a consolidação da humanização foi a melhoria da integração entre os setores e as equipes.

Os copeiros acabam sendo os parceiros nisso, porque como eles recolhem, eles veem a quantidade que voltou todos os dias [...]. Então eles interagem com os meninos no sentido: "Oh, tal paciente voltou um pouco mais" ou "voltou nada", então tem que colocar mais comida para ele (Nut1 - HPr).

A gente até é chamada, mas às vezes é no último momento. Podia ser mais, e não no último momento [...]. Outro dia teve um paciente que chamaram a ambulância, era até um paciente com sonda, e ele ainda precisava pegar a orientação do médico, precisava pegar a orientação com a gente e a ambulância ali esperando o paciente (Nut1 - HPu3).

A cooperação entre os profissionais da produção de refeições e problemas de comunicação com os demais profissionais do hospital também foram relatados. Estudo de Pedroso, Sousa \& Salles ${ }^{25}$ verificou que na prática de profissionais da saúde ocorriam predomínio de ações fragmentadas e não sistematizadas, além de dificuldades de interação com a equipe. Todavia, Pessini, Pereira \& Chanes ${ }^{40}$ salientam que a assistência integral só é possível na medida em que se consolida uma ação multiprofissional que busca a interdisciplinaridade.

Outro ponto levantado foram as condições de trabalho para a atuação profissional, destacando a necessidade do apoio institucional para investimentos em recursos humanos e materiais.

Ainda, a qualificação da equipe foi apontada como uma das iniciativas, envolvendo desde a dimensão nutricional e sensorial até os aspectos organizacionais do serviço.

Às vezes demora um pouco para qualificar o pessoal que chega, mas acreditamos que funcionários bem treinados são fundamentais (Nut2 - HPu3).

Nós temos um convênio com um curso de gastronomia e é interessante pra gente, porque eles nos oferecem um professor a cada semestre para dar um número "X" de aulas para os nossos funcionários. Então, isso é bastante interessante, acaba enriquecendo bastante o cardápio, a apresentação, de uma forma geral melhora muito a questão da aceitação da alimentação (Nut1 - HPr).

A dieta hipossódica, por exemplo, é uma bem rejeitada, então temos tentado trabalhar com outros temperos e com ervas pra deixar mais saboroso (Nut1 - HPu3).

Apesar de a educação permanente dos profissionais ser uma prioridade da $\mathrm{PNH},{ }^{16}$ esta iniciativa nos hospitais ainda não é freqüente. ${ }^{40}$ Para a implantação de ações humanizadoras, deve-se contar com pessoal sensibilizado, habilitado e com condições de trabalho. ${ }^{14,41}$ Portanto, é necessário entender a humanização considerando suas implicações institucionais, uma vez que 
não se trata apenas da relação pessoal entre profissional de saúde e paciente. ${ }^{18}$ Assim, quando se busca a humanização no contexto da PNH, é preciso pensar na valorização dos distintos sujeitos envolvidos no processo de produção da saúde (usuários, trabalhadores e gestores). ${ }^{16}$

As iniciativas propostas pelos nutricionistas apresentaram elementos para a humanização da atenção alimentar e nutricional ligada ao usuário e ao setor de produção de refeições, destacados abaixo, que se aproximam dos dispositivos propostos pela $\mathrm{PNH},{ }^{16}$ tais como a escuta qualificada e o projeto terapêutico singular; a educação permanente; a ambiência e a clínica ampliada e equipes de referência.

A escuta qualificada e o projeto terapêutico singular foram os dispositivos mais destacados, pelo reconhecimento de um cuidado alimentar que se aproxime das expectativas dos indivíduos e que ultrapasse a visão de doença e de satisfação de necessidades físicas e nutricionais, agregando o conforto e o respeito às preferências e aos hábitos alimentares. Além disso, a educação permanente esteve relacionada com os dispositivos acima, nos relatos sobre a necessidade de formação da equipe, envolvendo a dimensão nutricional e sensorial e a organização do serviço. A ambiência e a clínica ampliada e equipes de referência foram dispositivos pouco explorados. No entanto, reconheceram a necessidade de apoio institucional para investimentos em recursos humanos e materiais, e continuidade do tratamento para além do ambiente hospitalar, o que requer fortalecimento e diálogo com a rede social dos usuários.

No entanto, considerando as fragilidades da formação desses profissionais, que prioriza saberes técnico-científicos em detrimento de experiências e vivências intra e interpessoais, tão necessárias para a assistência humanizada, ${ }^{42}$ esses elementos necessitam de reflexões para seu fortalecimento e alinhamento com as políticas do Sistema Único de Saúde (SUS). Apesar de as diretrizes curriculares apontarem uma formação mais humanista, ${ }^{43}$ existe um grande caminho a trilhar, de aproximação com os cenários da prática e de uma nova práxis de fazer saúde. ${ }^{42,44}$

Destaca-se, por fim, que estas iniciativas de humanização da alimentação e nutrição são limitadas a grupos de nutricionistas inseridos em contextos de hospitais de um município. Portanto, não se referem necessariamente a iniciativas implementadas nas instituições em que atuam e somente podem ser analisadas como elementos de comparação e análise para outros grupos de profissionais dentro de outros contextos institucionais.

A utilização do Guia de Entrevistas pode ter limitado as iniciativas apontadas, mas tais achados podem servir de reflexão para futuras pesquisas, considerando a carência de estudos que relacionem alimentação hospitalar e práticas de humanização no Brasil. 


\section{Considerações finais}

As informações sobre a humanização do atendimento da alimentação hospitalar ainda são escassas, e a PNH apresenta propostas de ações generalistas, cabendo a cada instituição buscar, dentro de suas características, a adequação aos objetivos dessa política.

Na presente pesquisa, a percepção da humanização da alimentação hospitalar envolveu a individualização do atendimento considerando dimensões sensoriais, simbólicas e a qualificação do serviço (a exemplo do envolvimento de copeiros e uso de refeitórios). As iniciativas de humanização destacadas pelos nutricionistas envolveram aspectos relacionados às necessidades dos usuários e dos profissionais: educação nutricional; atendimento continuado após a alta hospitalar; melhoria nutricional e sensorial dos cardápios, conjugada à dimensão simbólica e convivial da alimentação; organização do serviço; ações educativas, sensibilização e valorização dos profissionais.

As iniciativas levantadas pelos nutricionistas foram percebidas como essenciais para a implementação de ações de alimentação e nutrição dentro do contexto de humanização. O conjunto dessas ações requer interação dos profissionais envolvidos, apoio institucional e individualização da atenção.

\section{Referências}

1. Westergren A, et al. Malnutrition prevalence and precision in nutritional care differed in relation to hospital volume: a cross-sectional survey. Nutr J [periódico na internet]. 2009 Maio [acesso em 2011 Ago 18];8(20)[aproximadamente 8 p.]. Disponível em: http://www.nutritionj.com/content/8/1/20 doi:10.1186/1475-2891-8-20

2. García de Lorenzo A, et al. Conclusiones del II Foro de Debate SENPE sobre desnutrición hospitalaria. Nutr Hosp. 2005; 20(2):82-7.

3. Gottraux S, et al. Screening and treatment of malnutrition: European Council Resolution and its potential application in Switzerland. Rev Med Suisse Romande. 2004;124(10):617-23.

4. Kondrup J, et al. Incidence of nutrition risk and causes of inadequate nutritional care in hospitals. Clin Nutr. 2002; 21(6):461-8.

5. Vecina NG, Malik AM. Tendências na assistência hospitalar. Ciên Saúde Colet. 2007; 12(4):825-39.

6. Thibault R, et al. Assessment of food intake in hospitalized patients: A 10-year comparative study of a prospective hospital survey. Clin Nutr. 2011; 30(3):289-96.

7. Wright ORL, Conelly LB, Capra S. Consumer evaluation of hospital foodservice quality: an empirical investigation. Int J Health Care Qual Assur Inc Leadersh Health Serv. 2006;19(2-3):181-94.

8. Lassen KO, Kruse F, Bjerrum M. Nutritional care of Danish medical inpatients: patients' perspectives. Scand J Caring Sci. 2005;19(3):259-67. 
9. Donini LM, et al. The quality of a restaurant service at a geriatric rehabilitation facility. Ann Ig. 2003;15(5):583-600.

10. Dupertuis YM, et al. Food intake in 1707 hospitalized patients: a prospective comprehensive hospital survey. Clin Nutr. 2003; 22(2):115-23.

11. Stanga Z, et al. Hospital food : a survey of patients' perceptions. Clin Nutr. 2003;22(3):241-6.

12. Brasil. Ministério da Saúde. Política Nacional de Humanização (Humaniza SUS) [homepage na Internet]. [acesso 2011 Set 06]. Disponível em: http://portal.saude.gov.br/portal/saude/cidadao/ area.cfm?id_area $=1342$

13. Barra DCC, et al. Evolução histórica e impacto da tecnologia na área da saúde e da enfermagem. Rev Eletr Enf [periódico na Internet]. 2006; 8(3):422-30. Disponível em: http://www.fen.ufg.br/revista/ revista8_3/v8n3a13.htm

14. Goulart BNG, Chiari BM. Humanização das práticas do profissional de saúde: contribuições para reflexão. Ciên Saúde Colet. 2010;15(1): 255-68.

15. Falk MLR, Ramos MZ, Salgueiro JB. A rede como estratégia metodológica da Política Nacional de Humanização: a experiência de um hospital universitário. Interface comun saúde educ. 2009;13(supl.1):709-17.

16. Brasil. Ministério da Saúde. Núcleo Técnico da Política Nacional de Humanização. HumanizaSUS: Política Nacional de Humanização: a humanização como eixo norteador das práticas de atenção e gestão em todas as instâncias do SUS. Brasília: Ministério da Saúde; 2004.

17. Deslandes SF. Análise do discurso oficial sobre a humanização da assistência hospitalar. Ciên Saúde Colet. 2004;9(1):7-14.

18. Ayres JRCM. Hermenêutica e humanização das práticas de saúde. Ciên Saúde Colet. 2005;10(3): 549-60.

19. Ayres JRCM. O cuidado, os modos de ser (do) humano e as práticas de saúde. Saúde soc. 2004;13(3):16-29.

20. Boff L. Saber cuidar: ética do humano: compaixão pela terra. Rio de Janeiro: Vozes; 1999.

21. Poulain JP, Saint-Sevin B. La restauration hospitalière. Toulouse, France: Cristal; 1990.

22. Poulain JP, Jeanneau S, Tibère L. Manger en milieu médicalisé: regards croisés, vers des applications concrètes. In: Ricour C. Mise en place d'une politique nutritionnelle Ministère de la Santé, de la Famille et des Personnes Handicapées. Décembre; 2002.

23. Sousa AA, Proença RPC. Tecnologias de gestão dos cuidados nutricionais: recomendações para a qualificação do atendimento nas unidades de alimentação e nutrição hospitalares. Rev Nutr. 2004; 17(4):425-36.

24. Demário RL, Sousa AA, Salles RK. Comida de hospital: percepções de pacientes em um hospital público com proposta de atendimento humanizado. Ciên Saúde Colet. 2010;15(supl. 1):1275-82.

25. Pedroso CGT, Sousa AA, Salles RK. Cuidado nutricional hospitalar: percepção de nutricionistas para atendimento humanizado. Ciên Saúde Colet. 2011;16(supl. 1):1155-62.

26. Minayo MCS. Pesquisa Social: Teoria, Método e Criatividade. 23. ed. Petrópolis: Vozes, 2004.

27. Flik U. Uma introdução à pesquisa qualitativa. 2. ed. Porto Alegre: Bookman, 2004. 
28. Sobal, J. Sample extensiveness in qualitative nutrition education research. J Nutr Educ. 2001; 33(4):184-92.

29. Diez-Garcia RW, et al. Gauging food and nutrition care quality in hospitals. Nutrition Journal. 2012;11;215-25.

30. Bardin L. Análise de conteúdo. Lisboa: Edições 70; 2004.

31. Garcia RWD. A dieta hospitalar na perspectiva dos sujeitos envolvidos em sua produção e em seu planejamento. Rev Nutr. 2006;19(2):129-44.

32. Gallian DMC. A desumanização do comer. Estud Ava. 2007; 21(60):179-84.

33. Godoy AM, Lopes DA, Garcia RWD. Transformações socioculturais da alimentação hospitalar. Hist. cienc. saude-Manguinhos. 2007;14(4):1197-215.

34. Santos LAS. Educação alimentar e nutricional no contexto da promoção de práticas alimentares saudáveis. Rev Nutr. 2005;18(5):681-92.

35. Bosi MLM, Uchimura KY. Avaliação da qualidade ou avaliação qualitativa do cuidado em saúde? Rev. Saúde Pública. 2007;41(1):150-3.

36. Erdmann AL, et al. Bioética e cuidados à saúde como responsabilidade com a vida e a natureza: algumas reflexões. Mundo Saúde. 2006;30(3):375-81.

37. Coloço RB, Holanda LB, Portero-Mclellan KC. Determinantes do grau de satisfação de pacientes internados referente a refeições oferecidas em um hospital universitário. Rev Ciênc Méd. 2009;18(3):121-30.

38. Deslandes S. O projeto ético-político da humanização: conceitos, métodos e identidade. Interface comun. saúde educ. 2005;9(17):389-406.

39. Benevides R, Passos E. A humanização como dimensão pública das políticas de saúde. Ciên. Saúde Colet. 2005;10(3): 561-71.

40. Pessini L, Pereira LL, Chanes M. Gestão em saúde: assistência integral, valorização da pessoa, investimentos crescentes. Mundo Saúde. 2006;30(2):197-202.

41. Leite TAAF, Strong MI. A influência da visão holística no processo de humanização hospitalar. Mundo Saúde. 2006;30(2):203-14.

42. Rede Unida. Estratégias para a implantação das diretrizes curriculares nacionais para os cursos de nutrição. In: Congresso Nacional da Rede Unida, V, 2003, Londrina. (Documento da Oficina 6).

43. Brasil. Câmara de Educação Superior. Resolução CNE/CES no 5, de 7 de novembro de 2001. Institui Diretrizes Curriculares Nacionais do Curso de Graduação em Nutrição. Diario Oficial da União. 09 nov 2001; Seção1:39. Disponível em: http://portal.mec.gov.br/cne/arquivos/pdf/CES05.pdf

44. Rossoni E, Lampert J. Formação de profissionais para o Sistema Único de Saúde e as diretrizes curriculares. Bol Saúde. 2004;18(1):87-98.

Recebido: 25/3/2013

Revisado: 05/6/2013

Aprovado: 21/7/2013 


\section{Apêndice A - Guia de Entrevistas}

Título da pesquisa: ALIMENTAÇÃO HOSPITALAR: ELEMENTOS PARA A CONSTRUÇÃO DE INICIATIVAS HUMANIZADORAS EM HOSPITAIS DE FLORIANÓPOLIS

\section{Perguntas norteadoras}

1. Como pode ser concebido um modelo de alimentação hospitalar humanizado para a Unidade de Alimentação e Nutrição?

2. Que atitudes humanizadoras são exequíveis para um cuidado alimentar e nutricional na Unidade de Alimentação e Nutrição?

\section{Guia de Entrevistas}

A) Construindo um modelo de alimentação hospitalar humanizado

- Para você, o que é uma alimentação hospitalar humanizada?

- Como é a realidade do trabalho da equipe de nutricionistas (o que se tem para desenvolver um trabalho qualificado)?

- Quais as suas necessidades como nutricionistas?

- Quais as necessidades dos pacientes hospitalizados?

B) Pensando em iniciativas humanizadoras

- Como pode ser conceituada a humanização na alimentação?

- Quais as condições de trabalho necessárias para o nutricionista?

- Comente algumas ações que podem ser consideradas humanizadoras:

- Avaliação, pelo paciente, do serviço oferecido pela equipe (apresentação, sabor, textura, horário e local onde são realizadas as refeições).

- Possibilidade de escolha de preparações fora do cardápio padrão.

- Ações de melhoria na qualidade sensorial (testes de novas receitas, uso de temperos, testes de degustação).

- Substituição ou complementação de alimentos após ingestão alimentar.

- Informações ao paciente e ações educativas. 